Recepción: 27 / 11 / 2017

Aceptación: 18 / 01 / 2018

Publicación: 21 / 03 / 2018

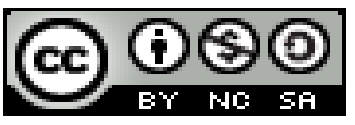

Ciencias de la salud

Artículo de Revisión

\title{
Urgencias periodontales en niños según factores de riesgo
}

\section{Periodontal emergencies in children according to risk factors}

\section{Emergências periodontais em crianças de acordo com fatores de risco}

\author{
Juan A. Oliveira-del Río ${ }^{\mathrm{I}}$ \\ juanoliveiradelrio@hotmail.com \\ Williams X. Buendía-Pizarro II \\ williams.buendia@hotmail.com \\ Freya M. Andrade-Vera III \\ freya.andrade@hotmail.com
}

Correspondencia: juanoliveiradelrio@hotmail.com

\begin{abstract}
I Magister Scientiarum em Odontopediatria, Doctor en Ciencias Odontológicas, Odontólogo, Docente de la Universidad Laica "Eloy Alfaro” de Manabí, Manta, Ecuador.

II Odontólogo, Docente de la Universidad Laica “Eloy Alfaro” de Manabí, Manta, Ecuador.

III Especialidad en Periodoncia, Odontóloga, Docente de la Universidad Laica "Eloy Alfaro" de Manabí, Manta, Ecuador.
\end{abstract}




\title{
Resumen
}

Se realizó un estudio descriptivo y transversal de 90 pacientes con manifestaciones clínicas de urgencias periodontales, atendidos en la consulta odontológica, desde junio del 2016 hasta junio del 2017. Entre las variables analizadas figuraron: grupos etarios, factores de riesgo y urgencias periodontales. En la serie $72.2 \%$ de los pacientes atendidos pertenecían al sexo masculino y $27.7 \%$ al femenino, el grupo de 12-14 años prevaleció con un 84.4\%, la Estomatitis aftosa fue la urgencia periodontal más frecuente $(41.1 \%)$, con respeto a los factores de riesgos la higiene bucal deficiente represento el $90.0 \%$. Se recomendó efectuar estudios en diferentes poblaciones para llevar a cabo acciones de promoción de salud y prevención de estas patologías.

Palabras clave: urgencias; enfermedades periodontales; factor de riesgo.

\begin{abstract}
A descriptive and cross-sectional study of 90 patients with clinical manifestations of periodontal emergencies was carried out, attended in the dental office, from June 2016 to June 2017. Among the variables analyzed were: age groups, risk factors and periodontal emergencies. In the series $72.2 \%$ of the patients attended belonged to the male sex and $27.7 \%$ to the female, the group of 12-14 years prevailed with $84.4 \%$, the aphthous stomatitis was the most frequent periodontal urgency $(41.1 \%)$, with respect to the risk factors deficient oral hygiene accounted for $90.0 \%$. It was recommended to carry out studies in different populations to carry out actions of health promotion and prevention of these pathologies.
\end{abstract}

Keywords: emergencies; periodontal diseases; risk factor.

\section{Resumo}

Foi realizado estudo descritivo e transversal de 90 pacientes com manifestações clínicas de emergências periodontais, atendidos no consultório odontológico, no período de junho de 2016 a junho de 2017. Entre as variáveis analisadas foram: faixas etárias, fatores de risco e emergências periodontais. Na série $72,2 \%$ dos pacientes atendidos pertenciam ao sexo masculino e $27,7 \%$ ao feminino, o grupo de 12-14 anos predominou com 84,4\%, a estomatite aftosa foi a urgência periodontal mais frequente $(41,1 \%)$, com relação à fatores de risco higiene bucal deficiente representaram 90,0\%. Recomenda-se a realização de estudos em diferentes populações para realizar ações de promoção da saúde e prevenção dessas patologias. 
Palavras chave: emergências; doenças periodontais; fator de risco.

\section{Introducción}

El término Enfermedad Periodontal (EP) abarca un conjunto de alteraciones que afectan a los tejidos que protegen y sostienen al diente: encía, ligamento alveolo-dentario, hueso alveolar y cemento radicular. Generalmente comienza con una inflamación de la encía (gingivitis) y puede continuar o no con la formación de bolsas y destrucción de tejidos profundos (periodontitis). La movilidad y pérdida de dientes caracterizan la etapa final. ${ }^{1}$

Las enfermedades gingivales y periodontales están catalogadas entre las afecciones más comunes del género humano. La gingivitis afecta aproximadamente al $80 \%$ de los niños de edad escolar, y más del $70 \%$ de la población adulta ha padecido de gingivitis, periodontitis o ambas. Los resultados de investigaciones y estudios clínicos revelan que las lesiones producidas por las periodontopatías en las estructuras de soporte de los dientes en los adultos jóvenes, son irreparables y que, en la tercera edad, destruye gran parte de la dentadura natural, privando a muchas personas de todos sus dientes durante la vejez. ${ }^{2-3}$

La prevalencia y gravedad de las periodontopatías varía en función de factores sociales, ambientales, enfermedades bucales y generales, y particularmente de la situación de higiene bucal individual. Los primeros signos de periodontopatías suelen ser evidentes después del segundo decenio de la vida y es común observar destrucciones considerables después de los 40 años. ${ }^{4}$

Las urgencias estomatológicas, representan un capítulo importante dentro del contexto general de todas las urgencias. Son afecciones que provocan grandes molestias en los pacientes presentan un componente afectivo importante, y que en la mayoría de los casos no implican peligro para la vida, pero sí se requiere la atención inmediata del odontólogo. 5,6

Se conoce con el nombre de urgencias en periodoncia aquellas afecciones que por las molestias que producen al paciente o que, por sus posibles complicaciones, requieren de la atención inmediata del profesional (especialista u odontólogo general). Entre las principales urgencias periodontales encontramos la gingivitis úlceronecrotizante aguda (GUNA), y la estomatitis aftosa 
recurrente (EAR), las cuales provocan grandes molestias a la población infantil, juvenil y adulta. ${ }^{7,6}$

La Gingivitis Ulceronecrotizante Aguda (GUNA) es una enfermedad inflamatoria, destructiva y dolorosa que afecta la encía marginal y papilar y en menor frecuencia la encía adherida. Es una enfermedad que se caracteriza por su aparición repentina, frecuentemente después de una enfermedad debilitante y/o infección respiratoria aguda. ${ }^{1}$

Los signos y síntomas de la Gingivitis Ulceronecrotizante Aguda son dolor, sangramiento gingival, halitosis, sabor metálico, salivación abundante y viscosa y sensación de acuñamiento interdentario. Extra bucalmente, pueden observarse fiebre y malestar general, adenopatías, fatiga, cefaleas, insomnio, depresión, anorexia y alteraciones gastrointestinales. ${ }^{1}$

Todavía no se ha establecido una causa específica de la GUNA, actualmente se acepta que es una infección gingival oportunista aguda, causada por la placa dental bacteriana en pacientes debilitados, mal nutridos, inmunodeficientes por infecciones virales (VIH) y otros factores de riesgo sistémicos, incluyendo sueño inadecuado, inusual estrés, convalecencia reciente de enfermedades, alcoholismo y hábito de fumar. ${ }^{8}$

La estomatitis aftosa recurrente (EAR) es una lesión que se caracteriza por la aparición de vesículas redondeadas con bordes definidos que se rompen después de uno o dos días, formando úlceras redondeadas deprimidas, presentan una región central rojo-grisácea con una periferia elevada en forma de reborde. Las lesiones pueden aparecer en cualquier parte de la mucosa bucal. Se cura en pocos días, semanas o meses según los factores de riesgo que la desencadenen. Los ataques pueden repetirse periódicamente. ${ }^{9}$

Los factores de riesgo pueden estar presentes en todas las etapas de la vida, pero en nuestro trabajo, el objetivo son las edades entre 5-11 y 12-14 años Nos preocupan las condiciones biológicas, psicológicas y sociales que se asocian con el incremento de la propensión a desarrollar determinadas enfermedades o desviaciones de la salud en esta etapa de la vida, si no se conoce cómo se comportan los factores de riesgo de estas enfermedades. ${ }^{6}$

Nos propusimos en este trabajo caracterizar las urgencias periodontales en niños según factores de riesgo, que acudieron a la consulta Odontología en el periodo junio 2016 a junio 2017. Para 
posteriormente tomar decisiones en la promoción y prevención de ellas, así como en su tratamiento, lo cual deberá modificar su comportamiento.

\section{Metodología}

Se realizó una investigación aplicada de tipo observacional, descriptivo, de corte transversal, con vista a caracterizarlas urgencias periodontales en niños según factores de riesgo, en el periodo junio 2016 a junio 2017.

La población estudio estuvo conformada por 90 pacientes que acudieron a consulta por presentar algún proceso inflamatorio agudo del periodonto. Las variables utilizadas fueron: edad (5-11, 1214), sexo, urgencias periodontales tales como: GUNA, GEHA, estomatitis aftosa, absceso gingival o absceso periodontal y los factores de riesgo (higiene bucal deficiente, apiñamiento dentario, empaquetamiento de alimentos, hábito de fumar, enfermedades sistémicas).

Para diagnosticar con la mayor precisión posible la GUNA se consideró que siempredebía estar presente la lesión crateriforme, con al menos tres de los demás signos y síntomas señalados. ${ }^{6}$

Con vista a determinar la higiene bucal se aplicó el índice de higiene bucal (revisado), solo califica como registro para toda la boca una superficie dental: la que peor higiene presenta, ya sea por cálculo o por placa, la extensión de estos se determinará por visión directa y mediante el sondaje con un explorador.

\section{Escala de calificación:}

6: cálculo supra gingival que cubre más de dos tercios de la superficie dentaria o banda continúa de cálculo infra gingival.

5: cálculo supra gingival que reviste más de un tercio y menos de dos tercios de la superficie dentaria o pequeños puntos de cálculo infra gingival.

4: Cálculos supra gingival que cubre no más de un tercio de la superficie dentaria.

3: Placa que resguarda dos tercios de la superficie dentaria.

2: Placa que reviste más de un tercio y menos de dos tercios de la superficie dentaria.

1: Placa que cubre menos de un tercio de la superficie dentaria o presencia de manchas extrínsecas, manchas. 
0: No hay señales de placa, ni manchas. ${ }^{10}$

Se realizó una encuesta de conocimiento a los padres de los pacientes con el objetivo de determinar el nivel de conocimiento que poseían con a la salud bucal en estos grupos de edades.

La información fue recogida en un formulario personal mediante el cual se acopiaron los siguientes datos: nombre del paciente, edad, sexo, tipo de urgencia periodontal, factores de riesgo presentes y la aplicación del índice de higiene bucal simplificado de Green y Vermillion (buena o mala higiene bucal).

Los resultados se resumieron y se introdujeron en una base de datos Excel, se utilizaron medidas de resumen de estadística descriptiva para las variables cualitativas y el método que se utilizó fue el porcentual.

\section{Resultados}

De los 90 pacientes que acudieron a consultas con urgencias parodontales el $72.2 \%$ pertenecían al sexo masculino y $27.7 \%$ al femenino el grupo de $12-14$ prevaleció con un $84.4 \%$. (Tabla I)

Tabla I. Distribución de pacientes afectados por urgencias periodontales según edad y sexo.

\begin{tabular}{|l|c|c|c|c|c|c|}
\hline \multirow{3}{*}{ Edad } & \multicolumn{4}{|c|}{ Sexo } & \multicolumn{2}{c|}{ Total } \\
\cline { 2 - 7 } & \multicolumn{2}{|c|}{ Masculino } & \multicolumn{2}{c|}{ Femenino } & \multicolumn{2}{|c|}{} \\
\cline { 2 - 7 } & No & $\%$ & No & $\%$ & No & $\%$ \\
\hline $5-11$ & 10 & 15.3 & 4 & 16 & 14 & 15.5 \\
\hline $12-14$ & 55 & 84.6 & 21 & 40 & 76 & 84.4 \\
\hline Total & 65 & 72.2 & 25 & 27.7 & 90 & 100 \\
\hline
\end{tabular}

Obsérvese en la tabla II, el mayor número de pacientes con urgencias periodontales estuvo representado por la Estomatis aftosa, 41 pacientes (45.5 \%) en el grupo de edad 12-14 años. El $56.3 \%$ de los niños atendidos presentaron rasgo de ansiedad. 
Tabla II. Urgencias periodontales según edad.

\begin{tabular}{|l|c|c|c|c|c|c|c|c|c|c|}
\hline \multirow{3}{*}{ Edad } & \multicolumn{9}{|c|}{ Urgencias } & \multicolumn{2}{c|}{ Total } \\
\cline { 2 - 11 } & $\begin{array}{c}\text { Estomatitis } \\
\text { aftosas }\end{array}$ & \multicolumn{2}{|c|}{ Absceso } & \multicolumn{2}{c|}{ GUNA } & \multicolumn{2}{c|}{ GEHA } & \multicolumn{2}{|c|}{} \\
\cline { 2 - 11 } & No & $\%$ & No & $\%$ & No & $\%$ & No & $\%$ & No & $\%$ \\
\hline $5-11$ & 1 & 2.4 & 2 & 16.6 & 2 & 9.0 & 1 & 6.6 & 6 & 6.6 \\
\hline $12-14$ & 40 & 97.5 & 10 & 83.3 & 20 & 90.9 & 14 & 40.0 & 84 & 93.3 \\
& & & & & & & & & & \\
\hline Total & 41 & 45.5 & 12 & 13.3 & 22 & 24.4 & 15 & 16.6 & 90 & 100 \\
\hline
\end{tabular}

La tabla III muestra los factores de riesgo presentes en los pacientes con urgencias periodontales, se apreció que el mayor número de ellos presentaron higiene bucal deficiente, con $81(90.0 \%)$ con predominio en el sexo masculino en $72.0(80.0 \%)$.

Tabla III. Factores de riesgo en pacientes con urgencias periodontales según sexo.

\begin{tabular}{|l|c|c|}
\hline Factores de riesgo & No & \% \\
\hline Higiene bucal deficiente & 81 & 90.0 \\
\hline Stress emocional & 28 & 31.1 \\
\hline Apiñamiento dentario & 42 & 46.6 \\
\hline Empaquetamiento de alimentos & 45 & 50 \\
\hline Diabetes mellitus & 6 & 6.6 \\
\hline Hábito de fumar & 2 & 2.0 \\
\hline
\end{tabular}

$\mathrm{Al}$ aplicar la encuesta a los padres de los pacientes y analizar la variable nivel de conocimiento acerca de la salud bucal, se obtuvo que solamente el $24.4 \%$ presentaron evaluación de adecuados el resto obtuvieron evaluación de inadecuado $75.5 \%$. 


\section{Discusión}

La salud bucal es parte integrante de la salud general, dado que un individuo no puede considerarse completamente sano, si existe presencia activa de enfermedad bucal. ${ }^{11}$

Los resultados alcanzados en la investigación, en relación con la distribución de pacientes afectados según edad, coinciden con el trabajo de Tamayo Márquez et al ${ }^{6}$ a pesar de que en su casuística estudio más grupos de edades.

Conforme a lo expuesto en otros estudios, en relación con las urgencias periodontales según edad, un gran número de autores coinciden en señalar la estomatitis aftosa como la más frecuente de las urgencias periodontales en este grupo de edad y resaltan que son lesiones extremadamente dolorosas. $^{12}$

El estudio coincidió con los resultados encontrados por Rojo17 sobre urgencias periodontales en una comunidad venezolana, donde la EAR constituyó $77,7 \%$ de las urgencias de este tipo encontradas en la población comprendida entre 12 y 18 años. ${ }^{1}$

La estomatitis o inflamación de la mucosa bucal es una infección viral frecuente en el niño, debido fundamentalmente al hábito que tienen de introducirse en la boca todo lo que encuentran. Es una enfermedad crónica, de carácter inflamatorio, que se caracteriza por la aparición de una o varias ulceras dolor osas en la mucosa oral, que persisten durante días o semanas y que residían tras periodos de remisión variables. ${ }^{13}$

Es importante reseñar que existen niños susceptibles de padecer estos procesos inflamatorios bucales, siendo la causa mal definida pero se barajan varias posibilidades como son un estado inmunitario deficiente por cualquier motivo, la presencia de estrés muchas veces condicionado por problemas escolares, pequeños traumatismos orales que se sobre infectan, la existencia de fluctuaciones hormonales, carencias vitamínicas, alergia a algún alimento o deficiencias nutricionales que pueden ocasionar que el niño padezca de forma reiterada esta patología. En nuestro estudio el $45.5 \%$ de los pacientes atendidos se le diagnostico esta patología.$^{13}$

Estudios realizados reflejan que la estomatitis aftosa se presenta a menudo en niños y adolescentes entre los 10 y 19 años. Las lesiones continúan apareciendo durante años después del 
brote inicial en un tercio de los niños afectados, no coincidió este resultado con los obtenidos en el trabajo. ${ }^{14}$

Una higiene inadecuada provoca acumulo de la placa dental bacteriana y provoca una respuesta inmune inflamatoria del huésped, lo que constituye el factor etiológico primario de la enfermedad periodontal junto con su componente microbiológico. ${ }^{15}$.

Los diabéticos presentan una serie de alteraciones en la respuesta de los tejidos periodontales a la acción de la placa dentobacteriana, de tal manera parece haber una mayor predisposición a padecer enfermedades periodontales más graves, con pérdida de los tejidos de soporte. En este proceso se piensa que es fundamental el control de la diabetes a la hora de valorar la progresión de la enfermedad periodontal, ya que, al parecer, los diabéticos mal controlados tienen una mayor tendencia a la hemorragia, a la pérdida de la inserción y a la pérdida del soporte óseo. En este sentido se establece un círculo vicioso, ya que a mejor control de los problemas periodontales de los pacientes, menores serán los requerimientos de insulina, por lo que se impone un control simultáneo de ambos procesos .Una vez identificado este factor de riesgo capaz de perpetuar o agravar esta enfermedad es que procederemos a actuar a través de la vigilancia estrecha de estos grupos y trazando estrategias capaces de reducir la morbimortalidad buco - dental. ${ }^{16}$ En la investigación existieron 6 pacientes con la enfermedad de diabetes mellitus correspondientes a las edades de 12-14-y 15-18.

Cupé-Araujo $\mathrm{A}^{17}$ plantea en su investigación que los padres desempeñan un papel crucial en el soporte emocional, necesidad de adaptación comportamental frente a enfermedades o tratamientos y en el financiamiento de los costos en la salud de los niños. Para que haya entonces salud bucal, los padres deben ser educados y concientizados para sensibilizar tempranamente a sus hijos en la adquisición de comportamientos que permitan un buen desenvolvimiento de hábitos de salud bucal.

Tomando en cuenta los niveles de conocimiento de los padres con respecto a la salud bucal, a pesar de que existen programas de promoción de salud el mensaje educativo no ha sido asimilado con la efectividad requerida.

Se concluyó que la estomatitis aftosa recurrente fue la urgencia periodontal más frecuente dentro de la población estudiada, la mayoría de las personas atendidas presentaron rasgo de ansiedad, los 
modos de afrontamiento al estrés más representados fue la búsqueda de apoyo social y los pacientes son vulnerables ante situaciones de estrés. Se encontró un predominio de la higiene bucal deficiente en todas las urgencias estudiadas.

\section{Referencias Bibliográficas}

1- Rojo Toledano M. Sosa Rosales M, Gandul Salabarria L. Comportamiento de Algunas Urgencias Periodontales en la Comunidad de "Betulio González. 2008 [citado 2 sep 2017]. Disponible en: https://www.portalesmedicos.com/publicaciones/articles/1238/1/Comportamiento-deAlgunas-Urgencias-Periodontales-en-la-Comunidad-de-Betulio-Gonzalez.html

2- Santos Peña M A el al. Urgencias Clínico Estomatológicas. Guías para el Diagnóstico. Rev. Cubana Estomatol. 2000; 37 (1): 5 - 49.

3- Cohen T L. Acute Necrotizing Ulcerative Epidemic. SADJ. 2002 Dec; 57 (11): 494.

4 Nazco Ríos C et al. Enfermedad Periodontal en Pacientes Infectados por el VIH. Rev. Cub. Estomatol. 2002; 40 (1): $17-23$.

5- Donado Rodríguez M. Urgencias en odontoestomatología. Madrid: Editorial. Madrid, abril; 1999. Crónica de la OMS. 21:6.

6- Martínez Lima J M, Sánchez Suárez O R, Lavandero Espina A, I Gonzalez del SolIV. L. Comportamiento de urgencias periodontales y factores de riesgo asociados en adolescentes, Policlínico "Ángel Ameijeiras", Guanabacoa. Revista Habanera de Ciencias Médicas 2014;13(2):196-206.

7- Colectivo de autores. Compendio de periodoncia. Tema III: Diagnóstico, pronóstico y tratamiento de la enfermedad periodontal inflamatoria aguda. Villa Clara: ISCM; 2006.

8 Blanco Berta L, Alfonso Calderón E. Comportamiento de la gingivitis ulceronecrotizante aguda: Departamento de estomatología de Conuco Viejo. Rev Med Electrón. 2010 [citado 2 septiembre 2017]; 32(3): Disponible en:http://scielo.sld.cu/scielo.php?script=sci_arttext\&pid=S168418242010000300004\&lng $=\mathrm{es}$ 
9 Sánchez PA: Manual de Periodontología. Universidad de Murcia. España: Ed Murcia; 2006.

10- Sosa Rosales M, Marin Quintero ME, Méndez Piña A, Guillaume Ramírez V, Criado M, Echarry Cano O. Indicadores Epidemiológicos En: Guías Prácticas de Estomatología. La Habana: Editorial Ciencias Médicas; 2003.p. 517-557.

11- Troya Borges Eddy, Martínez Abreu Judit, Padilla Suárez Ernesto, Iglesias López Nerelys, Ramos Ortega Annarais. La estomatitis aftosa recurrente y las situaciones de estrés como factor de riesgo. Rev. Med. Electron. [Internet]. 2014 Dic [citado 2017 Mayo]; 36(6): 799-812. Disponible en: http://scielo.sld.cu/scielo.php?script=sci_arttext\&pid=S1684$18242014000600001 \& \operatorname{lng}=\mathrm{es}$.

12- Pacho Saavedra JA, Piñol Jiménez FM. Estomatitis Aftosa Recurrente: Actualización. Rev Cubana Estomatol. 2005[citado 17 mayo 2017]; 42 (1): 34-75.Disponible en: http://scielo.sld.cu/scielo.php?script=sci_arttext\&pid=S0034-

13- Estomatitis aftosa recurrente en niños. Enciclopedia colaborativa Cubana EcuRed 2017 [citado 2 sep 2017]. https://www.ecured.cu/Estomatitis_aftosa_recurrente_en_ni\%C3\%B1os

14 Castillo Castillo A, Pérez Borrego A, Guntiñas Zamora MV. Uso del Inmunoferón en el tratamiento de la estomatitis aftosa recurrente en niños. Rev Cubana Estomatol. 2006 [citado 2 sep 2017]; 43(3) Disponible http://scielo.sld.cu/scielo.php?script=sci_arttext\&pid=S0034$75072006000300005 \& \operatorname{lng}=\mathrm{es}$

15- Elías Sierra N, González Longoria Ramírez YM. Respuesta inmune en enfermedad periodontal. RevInfCient. 2010 [citado 21 mayo 2017]; 65(1). Disponible en: http://bvscuba.sld.cu/?read_result=cumed-44025\&index_result=2

16- Marigio G Nicotine effects on polimohonuclear cell apoptosis and lipoplysacharideInduced monocyte functions. A Possible role in periodontal disease? J. Periodon. Res 2007; 36: 32-39. 
17- Cupé-Araujo A, García-Rupaya C. Conocimientos de los padres sobre la salud bucal de niños preescolares: desarrollo y validación de un instrumento. Rev Estomatol Herediana. 2015 Abr-Jun;25(2). 